\title{
CUIDADO MULTIPROFISSIONAL PARA O PACIENTE TERMINAL NA UTI: REVISÃO SISTEMÁTICA
}

\author{
MULTIPROFESSIONAL CARE IN TERMINAL ICU PATIENT: \\ SYSTEMATIC REVIEW
}

Thyara Batalha de Matos Gouveia', leda Maria Barbosa Aleluia'

Graduanda em Medicina na Escola Bahiana de Medicina e Saúde Pública. Salvador, Bahia, Brasil. http://orcid.org/0000-0002-9189-3347. thyaragouveia14.1@bahiana.edu.br ${ }^{2}$ Médica no Hospital da Sagrada Família. Doutora em Medicina e Saúde Humana. Docente na Escola Bahiana de Medicina e Saúde Pública e na Universidade do Estado da Bahia. Salvador, Bahia, Brasil. iedaleluia@bahiana.edu.br

RESUMO | introdução: A morte faz parte do ciclo vital e é um equívoco achar que o limite do cuidado é compatível com o limite terapêutico. No ambiente de unidade de terapia intensiva onde dispomos de diversas tecnologias para melhorar o estado funcional e até salvar a vida do paciente é necessário estabelecer os limites entre qualidade de vida e extensão da vida. A medicina paliativa surge neste ambiente com o objetivo de atender as demandas de todas as dimensões do cuidado ao paciente e a família, sendo este o papel da equipe multiprofissional. Objetivo: Avaliar o papel da equipe multiprofissional no cuidado do paciente terminal. Método: Trata-se de uma revisão sistemática. As buscas dos artigos foram realizadas no PubMed, na Biblioteca Virtual em Saúde (BVS) e na Scientific Eletronic Library Online Brazil (Scielo). Inicialmente foram lidos os títulos os artigos, depois os resumos e posteriormente a seleção foi a leitura na íntegra. Os artigos que foram selecionados foram aplicados de acordo com o critério PRISMA e o somatório da pontuação deveria ser maior que $70 \%$ dos itens para fazer parte do estudo. Resultados: Foram encontrados 864 artigos, que após a leitura dos títulos foram lidos 64 resumos, lidos na íntegra 29 artigos, foram para análise do prisma 12 e foram incluídos neste estudo 9. Destes, 2 eram revisões de literatura, 2 estudos multicêntricos, 1 pesquisa exploratório-descritiva, 1 estudo quanti-qualitativo, 1 estudo descritivo, 1 estudo de corte-transversal e 1 estudo randomizado Conclusão: A unidade de terapia intensiva é o ambiente onde a equipe multiprofissional é mais alinhada, porém quando o tema é cuidados paliativos a literatura demonstra que não ocorre de forma tão sincrônica. Os cuidados paliativos merecem maior atenção para que a morte seja conduzida de forma tranquila e digna.

Palavras-chave: Cuidado multiprofissional; Unidade de terapia intensiva; Cuidados paliativos; Paciente terminal; UTI.

\begin{abstract}
Introduction: Death is part of life cycle and is a mistake to think that care limit is consistent with therapeutic limit. In the intensive care unit environment where we can found any technologies to improve functional status and even save patient life is necessary to set limits between quality of life and length of life Palliative medicine comes up on this environment in order to reach all dimensions demand of patient and family care, being multidisciplinary team responsible for that. Objective: To evaluate the role of the multidisciplinary team in the terminal patient care. Method: This is a systematic review, searches of articles were performed in PubMed, the Virtual Health Library (BVS) and Scientific Electronic Library Online Brazil (Scielo). In the beginning the articles and titles were read, then resumed and then selection was completed ready. The items selected were applied in accordance with the PRISMA score criterion, and the sum should be greater than $70 \%$ of the items to be part of the study. Results: 864 articles were found, after select articles by title, 64 abstracts were rea. 29 articles were ready in full by Prisma analysis 12 and were included in Article 9 of these were 2 literature reviews, 2 multicenter, 1 exploratory research - descritive, 1 study quantitative and qualitative, descriptive study 1, 1 cross sectional studies and 1 randomized study. Conclusion: The intensive care unit is the environment where the multiprofessional team is more aligned, but when the subject is palliative care literature It demonstrates that there is not so synchronously. Palliative care deserve greater attention in order to conduct death in a peaceful and dignified manner.
\end{abstract}

Keywords: End-of-life; ICU; Profissional care; Care of death; Intensive care unit. 


\section{INTRODUÇÃO}

A unidade de terapia intensiva (UTI) é um local em que é possível utilizar a tecnologia para salvar a vida ou melhorar o estado funcional do paciente criando a impressão de poder aumentar o domínio sobre a morte e, desta forma, prorrogar a existência do enfermo. Porém, quando se trata de pacientes terminais, existe a necessidade de se estabelecer limites entre uma qualidade de vida melhor associada extensão da vida'.

A morte faz parte do ciclo vital e é um equívoco acreditar que não pode ser feito mais nada para - paciente que está fora da possibilidade de cura, pois enquanto há vida existe a necessidade do cuidado. Sendo dever da equipe multiprofissional prestar cuidados ao paciente durante todo processo, para que a morte ocorra de forma humanizada, além de proporcionar apoio aos familiares na vivência do luto².

A medicina paliativa surge como modalidade terapêutica que tem como filosofia melhorar a qualidade de vida dos pacientes, através da prevenção e do alívio dos sofrimentos físicos, psicossociais e espirituais. A assistência paliativa trata-se de uma abordagem complexa e objetiva que atende as demandas em todas as dimensões do cuidado ao paciente e a sua família, priorizando uma equipe multiprofissional que tenha como objetivo principal adotar uma postura reflexiva em relação às práticas de cuidado que visem à dignidade $\mathrm{e}$ totalidade do ser humano ${ }^{3}$.

O acolhimento da família e a proximidade do paciente com a equipe multiprofissional reconstituem uma existência inteira em todas as suas dimensões, buscando a plenitude de todo ser humano ao fim da vida. O cuidado aos familiares faz parte do cuidado global dos pacientes internados na UTI ${ }^{4}$. É necessário um cuidado terapêutico que englobe a equipe multidisciplinar adequadamente treinada, com o objetivo de identificar e diminuir os problemas no âmbito físico, psicológico, espiritual e social ${ }^{5}$.

Com o objetivo de permitir o alívio da angústia e da ansiedade, a equipe multiprofissional deve favorecer o desenvolvimento de um estado emocional tolerável e ser capaz de restaurar a estabilidade afetiva e as suas relações com o ambiente, além de permitir melhor aceitação da terminalidade. É importante discutir e verificar o cuidado aos assistidos no momento final da vida sob a ótica dos profissionais de saúde que trabalham na UTI, devido a necessidade do conforto ao paciente e do apoio aos familiares para que vivenciem este processo mais confortáveis e sem tanto sofrimento. Este artigo tem o objetivo de avaliar o papel da equipe multiprofissional no cuidado do paciente terminal, analisar a articulação da equipe multiprofissional diante do paciente terminal em relatos de literatura, além de descrever o conceito de cuidados paliativos.

\section{METÓDO}

\section{Desenho de estudo}

O estudo trata-se de uma revisão sistemática de literatura que é um método que sintetiza a produção científica disponível e que avalia criticamente e interpreta artigos relevantes disponíveis sobre um questionamento específico do conhecimento ${ }^{6}$.

\section{Ferramenta utilizada e estratégia de pesquisa}

A busca de artigos foi realizada através do PubMed, na Biblioteca Virtual em Saúde (BVS) e na Scientific Eletronic Library Online Brazil (Scielo), por meio da combinação de descritores/termos em ciências da saúde. Os termos utilizados foram "end-of-life", "ICU", "multidisciplinary care", "care of death", "intensive care unit", "cuidado multiprofissional", "unidade de terapia intensiva", "cuidados paliativos", "paciente terminal" e "UTI".

\section{Critérios de inclusão e exclusão}

Inclusão: Foram inclusos todos os trabalhos encontrados nas plataformas supracitadas através dos descritores que estivessem disponíveis na íntegra e gratuitamente, nos idiomas Português, Inglês e Espanhol. Os artigos utilizados foram publicados entre o ano de 2006 e 2016. 
Exclusão: Foram excluídos trabalhos que tivessem como abordagem principal a mortalidade de uma patologia específica e que o cuidado fosse apenas direcionado para a família.

\section{Identificação e seleção}

O pesquisador fez a leitura dos títulos dos trabalhos encontrados e foram incluídos apenas os que preenchiam os critérios de inclusão e que não preenchiam os critérios de exclusão. Após esta etapa foi realizada leitura dos resumos dos artigos pré-selecionados e verificado novamente se preenchia os critérios de inclusão e exclusão. Na terceira etapa, foi realizada a leitura na íntegra os artigos aprovados nas etapas anteriores com - objetivo de analisar se os critérios de inclusão e exclusão foram atendidos, seguida da análise metodológica dos artigos selecionados para serem utilizados na revisão sistemática.

\section{Avaliação metodológica dos artigos selecionados}

Os artigos foram avaliados segundo os critérios do PRISMA (Statement for Reporting Systematic Reviews and Meta-Analyses of Studies) que consiste em um check list com 27 itens. Os artigos selecionados foram avaliados e categorizados completando totalmente ou parcialmente cada item. Se o item foi abordado completamente recebia a pontuação 1 , se abordado parcialmente 0.5 e os itens não abordados recebiam 0 . Para fazer parte desta revisão sistemática o somatório da pontuação devia contemplar $70 \%$ dos itens (18.9). Foi confeccionada uma tabela que representa os artigos e suas respectivas pontuações (Tabela 1). Dois leitores procederam a esta análise.

Tabela 1. Comparativo das avaliações dos artigos baseado no PRISMA

\begin{tabular}{|c|c|c|c|c|c|c|c|}
\hline Tópicos & Itens & $\begin{array}{l}\text { Vicesi } \\
\text { MC } \\
(2016)\end{array}$ & $\begin{array}{l}\text { Tamaki } \\
\text { CM, } \\
\text { Meneguin } \\
\text { S, Alencar } \\
\text { RA, Luppi } \\
\text { CHB } \\
(2014)\end{array}$ & \begin{tabular}{|l|} 
Visser M, \\
Deliens L, \\
Houttekier \\
D (2014)
\end{tabular} & $\begin{array}{l}\text { Santos } \\
\text { MA, } \\
\text { Hormanez } \\
\text { M. (2013) }\end{array}$ & $\begin{array}{l}\text { Silva CF, } \\
\text { Souza } \\
\text { DM, } \\
\text { Pedreira } \\
\text { LC, } \\
\text { Santos } \\
\text { MR, } \\
\text { Faustino } \\
\text { TN } \\
(2013)\end{array}$ & $\begin{array}{l}\text { Pereira } \\
\text { MVG, } \\
\text { Freitas } \\
\text { NO } \\
(2013)\end{array}$ \\
\hline \multicolumn{8}{|l|}{ Título } \\
\hline Título & 1 & 0 & 0 & 1 & 1 & 0 & 0 \\
\hline \multicolumn{8}{|l|}{ Resumo } \\
\hline $\begin{array}{l}\text { Resumo } \\
\text { Estruturado }\end{array}$ & 2 & 0.5 & 0.5 & 1 & 0.5 & 1 & 1 \\
\hline \multicolumn{8}{|l|}{ Introdução } \\
\hline Racional & 3 & 1 & 1 & 1 & 1 & 1 & 1 \\
\hline Objetivos & 4 & 1 & 0.5 & 1 & 1 & 0.5 & 1 \\
\hline \multicolumn{8}{|l|}{ Métodos } \\
\hline $\begin{array}{ll}\text { Protocolo } & \text { e } \\
\text { registro }\end{array}$ & 5 & 0.5 & 0 & 0.5 & 0 & 0 & 0 \\
\hline $\begin{array}{l}\text { Critérios de } \\
\text { elegibilidade }\end{array}$ & 6 & 0 & 1 & 1 & 1 & 1 & 1 \\
\hline $\begin{array}{l}\text { Fontes de } \\
\text { informação }\end{array}$ & 7 & 0.5 & 1 & 1 & 1 & 1 & 1 \\
\hline Busca & 8 & 0 & 1 & 1 & 1 & 1 & 1 \\
\hline $\begin{array}{ll}\text { Seleção } & \text { dos } \\
\text { estudos } & \\
\end{array}$ & 9 & 0 & 1 & 1 & 1 & 1 & 1 \\
\hline $\begin{array}{l}\text { Processo de } \\
\text { coleta de dados }\end{array}$ & 10 & 0 & 1 & 1 & 1 & 1 & 1 \\
\hline Lista de dados & 11 & 1 & 1 & 1 & 1 & 1 & 1 \\
\hline $\begin{array}{l}\text { Risco de viés em } \\
\text { cada estudo }\end{array}$ & 12 & 0 & 0 & 0.5 & 0 & 0 & 0.5 \\
\hline $\begin{array}{l}\text { Medidas de } \\
\text { sumarização }\end{array}$ & 13 & 0 & 0 & 0.5 & 0 & 0.5 & 1 \\
\hline
\end{tabular}




\begin{tabular}{|c|c|c|c|c|c|c|c|}
\hline Tópicos & Itens & $\begin{array}{l}\text { Vicesi } \\
\text { MC } \\
(2016)\end{array}$ & $\begin{array}{l}\text { Tamaki } \\
\text { CM, } \\
\text { Meneguin } \\
\text { S, Alencar } \\
\text { RA, Luppi } \\
\text { CHB } \\
(2014)\end{array}$ & $\begin{array}{l}\text { Visser } M \text {, } \\
\text { Deliens L, } \\
\text { Houttekier } \\
\text { D (2014) }\end{array}$ & $\begin{array}{l}\text { Santos } \\
\text { MA, } \\
\text { Hormanez } \\
\text { M. (2013) }\end{array}$ & $\begin{array}{l}\text { Silva CF, } \\
\text { Souza } \\
\text { DM, } \\
\text { Pedreira } \\
\text { LC, } \\
\text { Santos } \\
\text { MR, } \\
\text { Faustino } \\
\text { TN } \\
(2013)\end{array}$ & $\begin{array}{l}\text { Pereira } \\
\text { MVG, } \\
\text { Freitas } \\
\text { NO } \\
\text { (2013) }\end{array}$ \\
\hline $\begin{array}{l}\text { Síntese dos } \\
\text { resultados }\end{array}$ & 14 & 1 & 1 & 1 & 0 & 1 & 1 \\
\hline $\begin{array}{l}\text { Risco de viés } \\
\text { entre estudos }\end{array}$ & 15 & 0 & 0 & 0.5 & 0 & 0 & 0 \\
\hline $\begin{array}{l}\text { Análises } \\
\text { adicionais }\end{array}$ & 16 & 0.5 & 1 & 1 & 1 & 1 & 1 \\
\hline \multicolumn{8}{|l|}{ Resultados } \\
\hline $\begin{array}{l}\text { Seleção de } \\
\text { estudos }\end{array}$ & 17 & 0 & 1 & 1 & 1 & 1 & 1 \\
\hline $\begin{array}{l}\text { Características } \\
\text { dos estudos }\end{array}$ & 18 & 1 & 0.5 & 1 & 1 & 1 & 1 \\
\hline $\begin{array}{l}\text { Risco de viés em } \\
\text { cada estudo }\end{array}$ & 19 & 0 & 0 & 0.5 & 0 & 0 & 0.5 \\
\hline $\begin{array}{l}\text { Resultados de } \\
\text { estudos } \\
\text { individuais }\end{array}$ & 20 & 1 & 1 & 1 & 1 & 1 & 1 \\
\hline $\begin{array}{ll}\text { Síntese } & \text { dos } \\
\text { resultados } & \end{array}$ & 21 & 1 & 0 & 1 & 1 & 1 & 1 \\
\hline $\begin{array}{l}\text { Risco de viés } \\
\text { entre estudos }\end{array}$ & 22 & 0 & 0 & 0.5 & 0 & 0 & 0.5 \\
\hline $\begin{array}{l}\text { Análises } \\
\text { adicionais }\end{array}$ & 23 & 1 & 1 & 1 & 1 & 1 & 1 \\
\hline \multicolumn{8}{|l|}{ Discussão } \\
\hline $\begin{array}{l}\text { Sumário da } \\
\text { evidência }\end{array}$ & 24 & 1 & 1 & 1 & 0.5 & 1 & 1 \\
\hline Limitações & 25 & 0 & 0.5 & 1 & 1 & 1 & 1 \\
\hline Conclusões & 26 & 1 & 1 & 1 & 1 & 1 & 1 \\
\hline \multicolumn{8}{|l|}{ Financiamento } \\
\hline Financiamento & 27 & 0 & 0 & 0 & 1 & 0 & 0 \\
\hline
\end{tabular}

Fonte: Elaborado pelo próprio autor, 2016.

\section{ANÁLISE DOS RESULTADOS}

\section{Identificação e seleção dos artigos}

A presente revisão de literatura identificou 3.965 artigos sobre o tema estudado. Foram reunidos 3.965 artigos pela estratégia de busca traçada nos bancos de dados disponíveis (Tabela 2). 


\begin{tabular}{c|c}
\hline BANCOS DE PESQUISA & NÚMERO DE ARTIGOS \\
\hline BVS & 3.436 \\
\hline PubMed & 504 \\
\hline Scielo & 25 \\
\hline Total & 3.965 \\
\hline
\end{tabular}

Fonte: Elaborado pelo próprio autor, 2016.

Ao realizar a busca na Biblioteca Virtual em Saúde foram utilizados os seguintes descritores "care of death and intensive care unit" e foram disponibilizados 3.436 artigos que foram filtrados de acordo com o critério de inclusão: idiomas em Português, Espanhol e Inglês, textos disponíveis na íntegra, publicados entre o ano de 2005 e 2012, colocado como assunto principal terapia intensiva, cuidados críticos, assistência terminal, cuidados paliativos, estado terminal e assunto da revista terapia intensiva. Como demonstrado na tabela 3 , após os filtros foram disponibilizados 779 artigos que seguiram para etapa de leitura do título. Ao pesquisar com os descritores "cuidado multiprofissional and unidade de terapia intensiva and cuidados paliativos and paciente terminal" foram disponibilizados 2 artigos que eram repetidos.

No PubMed, foram utilizados os descritores "end-of-life and ICU and profissional care" e foram disponibilizados 504 artigos. Após a utilização dos filtros: disponibilidade do texto na íntegra, publicação entre os 10 últimos anos e trabalhos com seres humanos, ficaram disponíveis 58 artigos que seguiram para a próxima etapa.

A busca no Scielo os descritores foram "paciente and terminal and UTI" foram disponibilizados 15 artigos e ao utilizar os termos "cuidados and paliativos and UTI" ficaram disponíveis 10, sendo 4 repetidos da busca anterior e 1 repetido da BVS.

Tabela 3. Análise dos bancos de pesquisa

\begin{tabular}{c|c|c}
\hline BANCOS DE PESQUISA & NÚMERO DE ARTIGOS & ARTIGOS REPETIDOS \\
\hline BVS & 781 & 2 \\
\hline Pubmed & 58 & 5 \\
\hline Scielo & 25 & 0 \\
\hline Total & 864 & 5 \\
\hline
\end{tabular}

Fonte: Elaborado pelo próprio autor, 2016.

Após a leitura dos títulos dos artigos encontrados na BVS foram selecionados 46 artigos, no PubMed foram 8 artigos e, no Scielo, 10 artigos seguindo os critérios de inclusão e exclusão, totalizando 64 artigos para a etapa seguinte de leitura dos resumos. Destes 64 artigos que foram selecionados para a leitura dos resumos foram selecionados 29 artigos para a próxima etapa que é a leitura completa do artigo. Após a leitura completa dos artigos foram selecionados 12 que seguirão para análise metodológica (Figura 1 ). 
Primeira etapa:

Busca de artigos

Artigos identificados nos bancos de dados $(n=864)$

Artigos excluídos após leitura do titulo

$$
(n=794)
$$

Artigos selecionados para leitura do resumo

$$
\text { ( } n=64)
$$

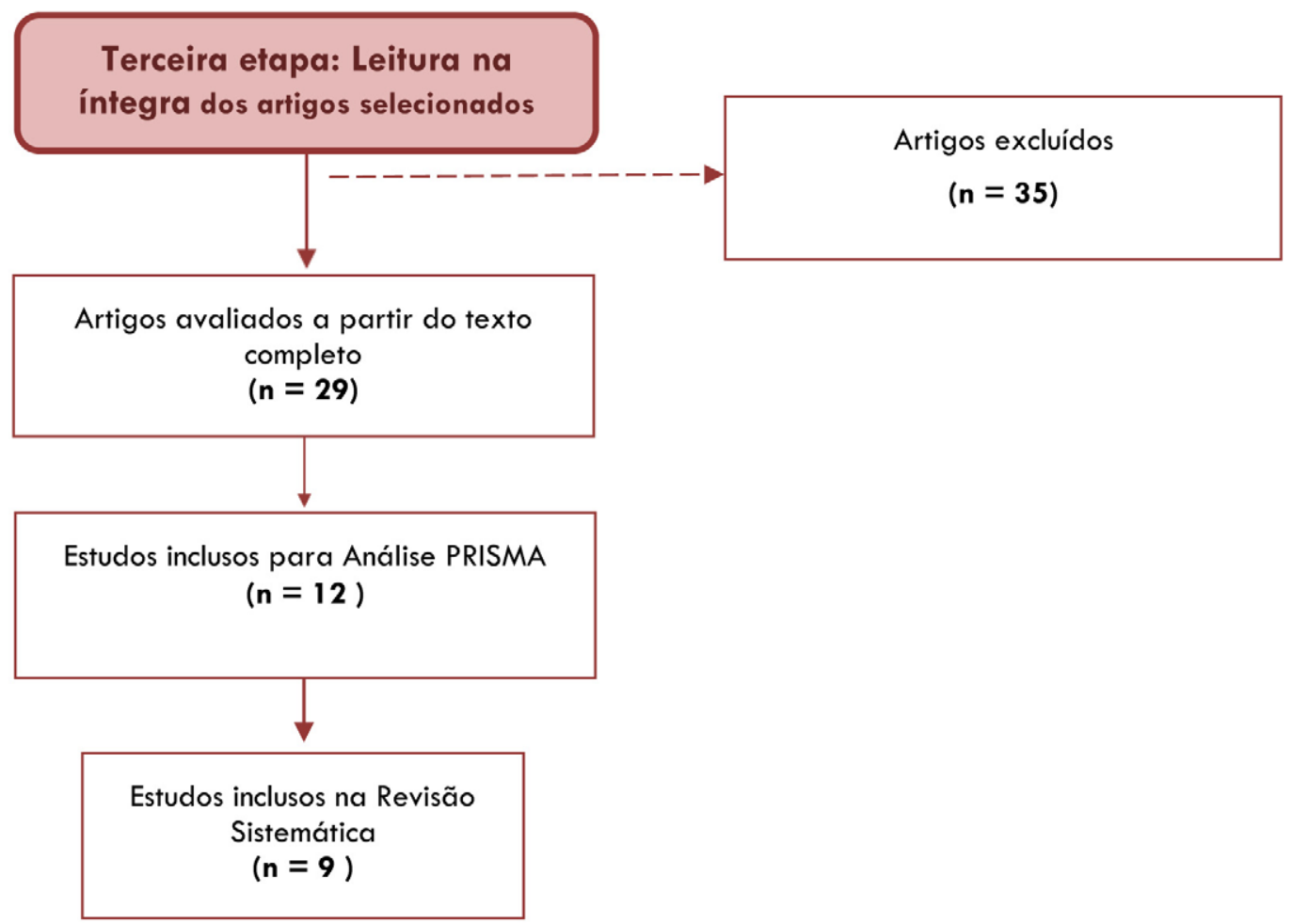

Figura 1- Fluxograma do processo de seleção dos estudos 
Após a leitura dos 12 artigos na íntegra e análise metodológica segundo os critérios definidos pelo PRISMA, verificou-se que nove artigos contemplaram de forma satisfatória. Sendo a porcentagem mínima de satisfação $70 \%$ de qualidade, foi construída uma tabela contemplando a pontuação de cada artigo seguindo a ordem do ano de publicação (Tabela 4).

Tabela 4. Pontuação total dos artigos de acordo com PRISMA

\begin{tabular}{|c|c|c|c|c|}
\hline AUTORES & $\begin{array}{l}\mathrm{N}^{\circ} \text { de itens } \\
\text { contemplados } \\
\text { totalmente }\end{array}$ & $\begin{array}{c}\mathrm{N}^{\circ} \text { de itens contemplados } \\
\text { parcialmente }\end{array}$ & $\begin{array}{l}\mathrm{N}^{\circ} \text { de itens não } \\
\text { contemplados }\end{array}$ & $\%$ \\
\hline Vicesi MC (2016) & 10 & 4 & 13 & $44.44 \%$ \\
\hline $\begin{array}{c}\text { Tamaki CM, } \\
\text { Meneguin S, Alencar } \\
\text { RA, Luppi CHB } \\
\text { (2014) }\end{array}$ & 14 & 4 & 9 & $59.25 \%$ \\
\hline $\begin{array}{l}\text { Visser M, Deliens L, } \\
\text { Houttekier D (2014) }\end{array}$ & 20 & 6 & 1 & $85.18 \%$ \\
\hline $\begin{array}{c}\text { Santos MA, } \\
\text { Hormanez M (2013) }\end{array}$ & 18 & 2 & 7 & $70.37 \%$ \\
\hline $\begin{array}{c}\text { Silva CF, Souza DM, } \\
\text { Pedreira LC, Santos } \\
\text { MR, Faustino TN } \\
(2013) \\
\end{array}$ & 18 & 2 & 7 & $70.37 \%$ \\
\hline $\begin{array}{c}\text { Pereira MVG, Freitas } \\
\text { NO }(2013)\end{array}$ & 20 & 3 & 4 & $79.62 \%$ \\
\hline $\begin{array}{c}\text { Ho LA, Engelberg } \\
\text { RA, Curtis JR, Nelson } \\
\text { J, Luce J, Ray DE et } \\
\text { al (2011) }\end{array}$ & 22 & 3 & 2 & $87.03 \%$ \\
\hline $\begin{array}{l}\text { Curtis JR, Nielsen EL, } \\
\text { Treece PD, Downey } \\
\text { L, Dotolo D, Shannon } \\
\text { SE et al (2011) } \\
\end{array}$ & 22 & 3 & 2 & $87.03 \%$ \\
\hline $\begin{array}{c}\text { Batista KT; Seidl EMF } \\
(2011)\end{array}$ & 20 & 2 & 5 & $77.77 \%$ \\
\hline $\begin{array}{c}\text { Moritz RD, Deicas A, } \\
\text { Rossini JP, Silva NB, } \\
\text { Lago PM, Machado } \\
\text { FO (2010) }\end{array}$ & 19 & 1 & 7 & $72.22 \%$ \\
\hline $\begin{array}{c}\text { Sulzbacher M, Reck } \\
\text { AV, Stum EMF, } \\
\text { Hildbrand LM (2009) }\end{array}$ & 17 & 2 & 8 & $66.66 \%$ \\
\hline $\begin{array}{l}\text { Glavan BJ, Downey } \\
\text { L, Curtis JR (2008) }\end{array}$ & 20 & 5 & 2 & $83.33 \%$ \\
\hline
\end{tabular}

Fonte: Elaborado pelo próprio autor, 2016.

\section{Características gerais dos estudos selecionados}

A Tabela 5 descreve, resumidamente, os artigos trazendo os objetivos e resultados com o propósito de sistematizar o conhecimento que será abordado adiante sobre a estratégia trazida nesta revisão de literatura sobre a equipe multiprofissional diante de um paciente terminal. 


\begin{tabular}{|c|c|c|c|c|}
\hline Autores & Tíłulo do artigo & $\begin{array}{l}\text { Ano } \\
\text { de } \\
\text { public } \\
\text { ação }\end{array}$ & Objetivos & Resultados \\
\hline $\begin{array}{l}\text { Visser M, Deliens L, } \\
\text { Houttekier D }\end{array}$ & $\begin{array}{l}\text { Physician- related } \\
\text { barriers to } \\
\text { communication and } \\
\text { patient- and family- } \\
\text { centred decision- } \\
\text { making towards the } \\
\text { end of life in intensive } \\
\text { care: a systematic } \\
\text { review }\end{array}$ & 2014 & $\begin{array}{l}\text { Descrever as barreiras } \\
\text { existentes na comunicação } \\
\text { entre o médico e a equipe, } \\
\text { além da comunicação do } \\
\text { médico com paciente e } \\
\text { família. }\end{array}$ & $\begin{array}{l}\text { As dificuldades de } \\
\text { comunicação encontradas } \\
\text { estão relacionadas com a } \\
\text { falta de confiança do } \\
\text { médico. }\end{array}$ \\
\hline $\begin{array}{l}\text { Santos MA, } \\
\text { Hormanez M }\end{array}$ & $\begin{array}{l}\text { Atiłude frente à morłe } \\
\text { em profissionais e } \\
\text { esłudantes de } \\
\text { enfermagem: revisão } \\
\text { da produção científica } \\
\text { da última década }\end{array}$ & 2013 & $\begin{array}{l}\text { Investigar atitude frente à } \\
\text { morte dos profissionais de } \\
\text { enfermagem. }\end{array}$ & $\begin{array}{l}\text { O assunto morte e morrer } \\
\text { tem sido negligenciado na } \\
\text { formação do profissional } \\
\text { o que acarreta } \\
\text { dificuldades e sofrimentos. }\end{array}$ \\
\hline $\begin{array}{l}\text { Silva CF, Souza DM, } \\
\text { Pedreira LC, Santos } \\
\text { MR, Faustino TN }\end{array}$ & $\begin{array}{l}\text { Concepções da equipe } \\
\text { multiprofissional sobre } \\
\text { a implementação dos } \\
\text { cuidados paliativos na } \\
\text { unidade de terapia } \\
\text { intensiva }\end{array}$ & 2013 & $\begin{array}{l}\text { Analisar a concepção da } \\
\text { equipe multiprofissional } \\
\text { diante de cuidados } \\
\text { paliativos na UTI adulto. }\end{array}$ & $\begin{array}{l}\text { Os cuidados paliativos } \\
\text { são negligenciados pela } \\
\text { equipe multiprofissional }\end{array}$ \\
\hline $\begin{array}{l}\text { Freitas NO, Pereira } \\
\text { MVG }\end{array}$ & $\begin{array}{l}\text { Percepção dos } \\
\text { enfermeiros sobre } \\
\text { cuidados paliativos e o } \\
\text { manejo da dor na UTI }\end{array}$ & 2013 & $\begin{array}{l}\text { Analisar a percepção do } \\
\text { enfermeiro que trabalha na } \\
\text { UTI sobre cuidados } \\
\text { paliativos. }\end{array}$ & $\begin{array}{l}\text { A ausência dos cuidados } \\
\text { paliativos ocorre devido } \\
\text { falta de conhecimento dos } \\
\text { profissionais. }\end{array}$ \\
\hline $\begin{array}{l}\text { Ho LA, Engelberg } \\
\text { RA, Curtis JR, } \\
\text { Nelson J, Luce J, } \\
\text { Ray DE et al }\end{array}$ & $\begin{array}{l}\text { Comparing Clinician } \\
\text { Ratings of the Quality } \\
\text { of Palliative Care in the } \\
\text { Intensive Care Unit }\end{array}$ & 2011 & $\begin{array}{l}\text { Avaliar a qualidade dos } \\
\text { cuidados paliativos de } \\
\text { médicos e enfermeiros na } \\
\text { UTI. }\end{array}$ & $\begin{array}{l}\text { Por passar mais tempo a } \\
\text { beira do leito, os } \\
\text { enfermeiros desempenham } \\
\text { maior cuidado paliativo } \\
\text { na UTI. }\end{array}$ \\
\hline $\begin{array}{l}\text { Curtis JR, Nielsen } \\
\text { EL, Treece PD, } \\
\text { Downey L, Dotolo } \\
\text { D, Shannon SE et al }\end{array}$ & $\begin{array}{l}\text { Effect of a Quality- } \\
\text { Improvement } \\
\text { Intervention on End-of- } \\
\text { Life Care in the } \\
\text { Intensive Care Unit }\end{array}$ & 2011 & $\begin{array}{l}\text { Avaliar uma intervenção } \\
\text { multidisciplinar na } \\
\text { assistência ao terminal na } \\
\text { UTI. }\end{array}$ & $\begin{array}{l}\text { Não houve nenhum efeito } \\
\text { sob a assistência dos } \\
\text { cuidados paliativos } \\
\text { direcionada a família. }\end{array}$ \\
\hline $\begin{array}{l}\text { Batista KT; Seidl } \\
\text { EMF }\end{array}$ & $\begin{array}{l}\text { Estudo acerca de } \\
\text { decisões éticas na } \\
\text { terminalidade da vida } \\
\text { em unidade de terapia } \\
\text { intensiva }\end{array}$ & 2011 & $\begin{array}{l}\text { Descrever conhecimentos e } \\
\text { condutas de médicos } \\
\text { intensivistas em situações de } \\
\text { limitação de suporte vital. }\end{array}$ & $\begin{array}{l}\text { Desconhecimento sobre o } \\
\text { tema. }\end{array}$ \\
\hline $\begin{array}{l}\text { Moritz RD, Deicas } \\
\text { A, Rossini JP, Silva } \\
\text { NB, Lago PM, } \\
\text { Machado FO }\end{array}$ & $\begin{array}{l}\text { Percepção dos } \\
\text { profissionais sobre o } \\
\text { tratamento no fim da } \\
\text { vida, nas unidades de } \\
\text { terapia intensiva da } \\
\text { Argentina, Brasil e } \\
\text { Uruguai }\end{array}$ & 2010 & $\begin{array}{l}\text { Avaliar condutas realizadas } \\
\text { nas UTIs diante de um } \\
\text { paciente terminal }\end{array}$ & $\begin{array}{l}\text { Nas UTIs do Brasil resistem } \\
\text { para iniciar os cuidados } \\
\text { paliativos }\end{array}$ \\
\hline $\begin{array}{l}\text { Glavan BJ, Downey } \\
\text { L, Curtis JR }\end{array}$ & $\begin{array}{l}\text { Using the Medical } \\
\text { Record to Evaluate the } \\
\text { Quality of End-of-Life } \\
\text { Care in the Intensive } \\
\text { Care Unit }\end{array}$ & 2008 & $\begin{array}{l}\text { Relacionar o prontuário } \\
\text { médico com a satisfação da } \\
\text { família na qualidade de } \\
\text { morte e morrer na UTI }\end{array}$ & $\begin{array}{l}\text { O prontuário não } \\
\text { consegue trazer toda } \\
\text { complexidade do cuidado } \\
\text { no fim da vida. }\end{array}$ \\
\hline
\end{tabular}


Os artigos utilizados compreendiam duas revisões de literatura, uma pesquisa exploratório-descritiva, um estudo quanti-qualitativo, dois estudos multicêntricos, um estudo-descritivo, um estudo de corte-transversal e um estudo randomizado (Tabela 6).

As revisões de literatura encontradas possuíam enfoques diferentes. Uma revisão trouxe a comunicação entre a equipe médica com a família, com o paciente e com a equipe multiprofissional. Foi realizada uma pesquisa em quatro bases de dados eletrônicas (MEDLINE, Embase, CINAHL e PsycINFO), foram utilizados 36 artigos que evidenciaram a falta de treinamento de comunicação e habilidade dos médicos frente a morte na UTI?. Enquanto que a outra revisão de literatura trouxe abordagem dos enfermeiros frente a morte. Os dados foram coletados por meio das bases de dados Lilacs, MEDLINE, PsycINFO e CINAHL, foram utilizados 35 artigos na íntegra que trouxe como principal evidência a falta de preparo dos profissionais desde a formação acadêmica para lidar com o processo de morte e morrer ${ }^{8}$.

Tabela 6. Tipo de estudo dos artigos utilizados

\begin{tabular}{|c|c|}
\hline Título do artigo & Tipo de estudo \\
\hline $\begin{array}{l}\text { Physician- related barriers to communication 'and patient- } \\
\text { and family-centred decision-making towards the end of life } \\
\text { in intensive care: a systematic review }{ }^{7}\end{array}$ & Revisão de literatura \\
\hline $\begin{array}{l}\text { Atitude frente à morte em profissionais e estudantes de } \\
\text { enfermagem: revisão da produção científica da última } \\
\text { década }^{8}\end{array}$ & Revisão de literatura \\
\hline $\begin{array}{l}\text { Concepções da equipe multiprofissional sobre a } \\
\text { implementação dos cuidados paliativos na unidade de } \\
\text { terapia intensiva }\end{array}$ & Pesquisa exploratório-descritiva \\
\hline $\begin{array}{l}\text { Percepção dos enfermeiros sobre cuidados paliativos e } 0 \\
\text { manejo da dor na UTI }\end{array}$ & Estudo quanti-qualitativo \\
\hline $\begin{array}{l}\text { Comparing Clinician Ratings of the Quality of Palliative Care } \\
\text { in the Intensive Care Unit }{ }^{10}\end{array}$ & Estudo multicêntrico \\
\hline $\begin{array}{l}\text { Effect of a Quality-Improvement Intervention on End-of-Life } \\
\text { Care in the Intensive Care Unit }{ }^{12}\end{array}$ & Estudo randomizado \\
\hline $\begin{array}{l}\text { Estudo acerca de decisões éticas na terminalidade da vida } \\
\text { em unidade de terapia intensiva }{ }^{13}\end{array}$ & Estudo descritivo \\
\hline $\begin{array}{l}\text { Percepção dos profissionais sobre o tratamento no fim da } \\
\text { vida, nas unidades de terapia intensiva da Argentina, Brasil } \\
\text { e Uruguai }{ }^{14}\end{array}$ & Estudo de corte-transversal \\
\hline $\begin{array}{l}\text { Using the Medical Record to Evaluate the Quality of End-of- } \\
\text { Life Care in the Intensive Care Unit"1 }\end{array}$ & Estudo multicêntrico \\
\hline
\end{tabular}

Fonte: Elaborado pelo próprio autor, 2016.

O artigo que foi realizado através de pesquisa exploratório-descritiva foi com quatro profissionais de saúde de um hospital público de grande porte em Salvador (BA). Os dados foram coletados entre fevereiro e abril de 2012 através de entrevista semiestruturada e observação e interpretação dos dados encontrados. Fizeram parte do estudo 6 enfermeiros, 4 fisioterapeutas, 3 médicos e 1 nutricionista. Este artigo aborda que os cuidados paliativos da equipe multiprofissional estão direcionados mais ao conforto físico e lesões físicas do que os cuidados relacionado a família e espiritualidade 5 . 
A pesquisa quanti-quantitativo foi realizada com enfermeiros que trabalhavam na UTI adultos de um hospital universitário em São Paulo e participaram oito enfermeiros 9 . A coleta foi realizada no período de junho a agosto de 2011 por meio de entrevistas. Foram utilizados instrumentos para coleta de dados quantitativos tais como sexo, idade, estado civil, religião, tempo de experiência e atuação na instituição. Para análise dos dados qualitativos os depoimentos foram transcritos e sistematizados?.

Um estudo multicêntrico fez análise em 13 hospitais nos Estados Unidos, os participantes foram 188 médicos intensivistas e 289 enfermeiros de cuidados críticos, foram dados escalas de $0-10$ para caracterizar a assistência do paciente terminal ${ }^{10}$. O outro estudo multicêntrico realizou um gráfico padronizado com levantamento de propostas de marcadores de qualidade do processo de morte e morrer na UTI pelas famílias de pacientes que morreram na UTI ou que no prazo de 24 horas depois de serem transferidos de uma UTI, foram aplicamos em UTIs de 10 hospitais no Noroeste dos Estados Unidos, em 356 pacientes ${ }^{11}$.

O artigo com o tipo de estudo randomizado foi realizado em 12 hospitais, com a intenção de avaliar a eficácia de uma intervenção de melhoria do processo de morte e morrer na UTI que tiveram como população de estudo a família dos pacientes que morreram na UTI ou no prazo de 30 horas após a alta da UTI usando pesquisas e revisão de prontuários. Foram realizados com 2.318 pacientes e os formulários eram encaminhados 72 horas após o óbito ${ }^{12}$.

O estudo descritivo contou com a utilização de um questionário anônimo e auto aplicado durante evento científico sobre terapia intensiva no CentroOeste do Brasil e participaram 15 médicos intensivistas. $O$ instrumento levantava dados sociodemográficos e profissionais como: idade, gênero, procedência, religião, tempo de formado, área de atuação e número de empregos, além de investigar o conhecimento sobre morte encefálica e ortotanásia $^{13}$.

O trabalho realizado pelos membros do grupo de estudo do final da vida das sociedades Argentina, Brasileira e Uruguaia de Terapia Intensiva foi do tipo corte-transversal. Eles elaboraram um questionário aplicado nos membros da equipe multiprofissional das sociedades, totalizando 420 profissionais. Este questionário constava de avaliações demográficas com os participantes, sobre as instituições em que trabalhavam e decisões sobre limites terapêuticos ${ }^{14}$.

\section{DISCUSSÃO}

Este estudo tipo revisão sistemática realizada com nove artigos teve como objetivo primordial avaliar o papel da equipe multiprofissional no cuidado do paciente terminal na UTI, descrevendo o conceito de cuidados paliativos e analisando a percepção da equipe multiprofissional em relatos de literatura.

O cuidado paliativo possui o objetivo de diminuir - sofrimento dos pacientes que possuem doença irreversível permitindo a manutenção da qualidade de vida do indivíduo e da sua família. É uma abordagem terapêutica que visa a redução dos problemas nas esferas psíquica, física, espiritual e social ${ }^{5}$.

O paliativismo tem como princípio proporcionar alívio da dor e conforto ao paciente em fase terminal da vida que devem ser ofertados juntamente com os cuidados curativos ${ }^{9}$. Batista e Seidl ${ }^{13}$ disseram que "os limites da medicina na preservação da vida e alívio do sofrimento são tênues e podem tornar-se antagônicos em algumas situações".

É fundamental a consolidação do cuidado paliativo como possível também no ambiente da UTI, por ser um direito do indivíduo e dever da equipe de saúde, tornando-se indispensável o atendimento qualificado de uma equipe multiprofissional. Pois, através dos múltiplos olhares é possível objetivar e assistir o paciente terminal em todas as dimensões, oferecendo bem-estar e respeitando a dignidade 5 .

Foi possível verificar em todos os artigos analisados - despreparo da equipe multiprofissional na UTI em lidar com o paciente terminal e os desafios da abordagem paliativa em ambiente de terapia intensiva ${ }^{5,7}, 8,9,10,11,12,13,14$.

Visser e cols. ${ }^{7}$ relataram que o desacordo e opiniões conflitantes sobre $\circ$ prognóstico do paciente $e$ 
tratamento na iminência da morte por diferentes médicos, dificulta o início abordagem paliativa, além de que algumas especialidades médicas eram mais resistentes para iniciar os cuidados paliativos. Normalmente, os cirurgiões são treinados para crer que o objetivo do tratamento é restrito a sobrevivência, e tendem a não aceitar que o paciente está morrendo?.

Visser e cols ${ }^{7}$, Silva e cols ${ }^{8}$, Batista e Seidl ${ }^{13}$, Moritz e cols 10 trouxeram fortes evidências que as crenças e os valores pessoais dos médicos podem dificultar o processo de criação e documentação sobre as metas dos cuidados para a família e para o paciente.

médico intensivista deve estar preparado para aceitação da limitação terapêutica e conduzir para abordagem paliativa que deve ser discutida com a equipe multiprofissional para a minimização do sofrimento durante este momento da vida $^{5,7,8,9,10,11,12,14}$. Moritz e cols ${ }^{14}$ mostraram que as características dos profissionais das UTIs apresentam diferenças relacionadas a cada país diferente. Foi constatado que a equipe médica é a principal responsável pela decisão do limite terapêutico, principalmente no Brasil e na Argentina, porém no Brasil é possível verificar maior participação dos outros setores envolvidos no cuidado ${ }^{13}$.

Os artigos realizados por enfermeiros relatam que a equipe médica não valoriza as diferenças disciplinares e não abrem espaço para a opinião de outros profissionais, o que gera insatisfação e desânimo por parte da equipe multiprofissional. Relatam também que a equipe médica possui dificuldade em notar que o ato de cuidar vai além da terapêutica curativa ${ }^{8,9}$. Ho e cols ${ }^{10}$ evidenciaram que os cuidados paliativos entregues aos enfermeiros para serem avaliados por enfermeiros eram significativamente maiores do que os cuidados dos médicos quando avaliados por médicos. Esta classificação foi determinada devido o papel do enfermeiro na UTI que gasta mais tempo à beira leito do que os médicos.

Curiosamente, Ho e cols ${ }^{10}$ mostraram que quando os cuidados paliativos da enfermagem eram avaliados, - resultado independia do avaliador, porém quando os cuidados paliativos dos médicos eram avaliados, o resultado modificava significativamente dependendo os avaliadores. Os médicos, quando avaliados por médicos, apresentavam maiores índices de cuidados paliativos, do que quando os enfermeiros classificavam os médicos ${ }^{10}$.

É válido ressaltar a importância do registro do cuidado em prontuário. Glavan e cols ${ }^{11}$ evidenciaram que a documentação do registro médico na presença de uma vida que se vai, associado com o desejo pessoal do paciente podem refletir em efeitos positivos associado ao planejamento de cuidados com o fim da vida do paciente e suas famílias. É sugerido que a documentação da avaliação da dor está associada a melhora da impressão da família na qualidade da experiência de morrer ${ }^{11}$.

O registro médico é um processo importante para a melhoria da prestação de cuidados, além de unificar o cuidado. É válido ressaltar que a ausência de registro em prontuário gera, a cada turno, uma nova conduta de acordo com o plantonista daquele período. Dessa forma, realizar o registro adequadamente conduz a assistência e permite a manutenção dos cuidados para a condução de uma morte digna $^{5,11}$.

Nestes estudos, foi possível observar que o atendimento aos pacientes terminais na unidade de terapia intensiva eram os últimos a serem atendidos, devido à atribuição da assistência para os pacientes com possibilidade de cura. Porém é importante discutir que há uma vida que necessita de cuidado da equipe multiprofissional que deve manter ao máximo o bem-estar deste paciente e proporcionar o melhor processo de morte possível ${ }^{5,7,9}$.

A morte digna é um processo dinâmico que envolve - paciente, a família, cuidadores e profissionais, no qual os cuidados físicos são cruciais, mas também os

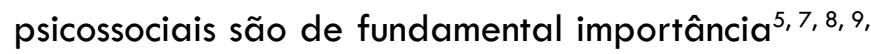
$10,11,12,13,14$. É possível notar altas prevalências de sintomas de ansiedade, depressão e transtorno de estresse pós-traumático em familiares de pacientes na UTI com quadro terminal ${ }^{12}$.

Curtis e cols ${ }^{12}$ evidenciaram que a comunicação mais cedo é eficaz para aceitação dos pacientes e familiares sobre os cuidados de fim de vida e que pode resultar em uma melhor atenção para minimizar os tratamentos que promovem prolongamentos desnecessários, melhorando a qualidade de morte. 
Os cuidados terapêuticos devem ser concomitantes com os cuidados paliativos, pois previne o sofrimento do paciente e dos seus familiares $5,7,8,9,10,11,12,13,14$. É essencial a informação de um prognóstico honesto para os pacientes e familiares para que os mesmos tenham tempo para preparar e elaborar o luto ${ }^{15}$.

Diante do abordado nos artigos encontrados e na literatura se faz necessária a criação de protocolos de cuidados paliativos nos hospitais com unidades de terapia intensiva, além da necessidade de introdução da disciplina nas universidades $5,7,8,9,10,11,12,13,14$.

As limitações deste artigo envolve a precariedade de estudos que englobem a visão da equipe multiprofissional, já que grande parte dos artigos ou estão envolvidos com a definição de cuidados paliativos e sob a ótica de profissionais específicos. Sendo necessário, desta forma, realizar comparação entre os profissionais de maneira isolada.

\section{CONCLUSÃO}

Diante deste estudo foi possível concluir que ainda se tem muito o que avançar em termos de cuidados paliativos, principalmente na unidade de terapia intensiva. Apesar de ser o ambiente do hospital em que a equipe multiprofissional funciona mais alinhada, é relatado que não evolui o paciente terminal como deveria, e que a ausência de protocolos que orientem estes profissionais para a decisão e início da terapêutica paliativa tem um papel importante na condução do paciente e aceitação da família.

É de suma importância entender que o paciente terminal envolve diversas esferas tanto física, quanto psíquica e social, além de possuir uma família que sofre juntamente e aguarda uma recuperação que nem sempre é possível de acontecer, já que a morte faz parte do ciclo da vida.

Os cuidados paliativos merecem uma maior atenção para que a morte seja conduzida de forma tranquila e digna, que os cuidados continuem sendo ofertados mesmo quanto a terapêutica não seja mais eficiente. A equipe multiprofissional tem o dever de conduzir da melhor forma a morte do paciente e aceitação da família, permitindo que ocorra de maneira humana e natural.

\section{AGRADECIMENTOS}

Agradeço imensamente a Dra leda Aleluia, professora da Escola Bahiana de Medicina e Saúde Pública, por todo apoio e dedicação para a realização deste artigo. Agradeço também a Dra Hermila Guedes que me auxiliou na construção do trabalho de conclusão de curso, fonte de inspiração para a produção deste artigo, e a Dra Tarita Batalha por ser minha fonte de inspiração profissional.

\section{COMPETING INTERESTS}

Nenhum conflito financeiro, legal ou político envolvendo terceiros (governo, empresas e fundações privadas, etc.) foi declarado para nenhum aspecto do trabalho submetido (incluindo mas não limitandose a subvenções e financiamentos, conselho consultivo, desenho de estudo, preparação de manuscrito, análise estatística, etc).

\section{REFERÊNCIAS}

1. Chaves AAB, Massarollo MCKB. Percepção de enfermeiros sobre dilemas éticos relacionados a pacientes terminais em unidades de terapia intensiva. Rev Esc Enferm USP. 2009;43(1):30-6. doi: 10.1590/S0080$\underline{62342009000100004}$

2. da Silva RS, Campos AER, Pereira A. Cuidando do paciente no processo de morte na unidade de terapia intensiva. Rev Esc Enferm. USP. 2011 ; 45(3):738-44. doi: $10.1590 /$ S0080-62342011000300027

3. Cardoso DH, Muniz RM, Schwartz E, Arrieira ICO. Cuidados paliativos na assistência hospitalar: a vivência de uma equipe multiprofissional. Texto Contexto Enferm. 2013;22(4):1 134141. doi: $10.1590 /$ S0104-07072013000400032

4. Soares M. Cuidando da família de pacientes em situação de terminalidade internados na unidade de terapia intensiva. Rev Bras Ter Intensiva. 2007;19(4):481-84. doi: 10.1590/ S0103-507X2007000400013

5. da Silva CF, Souza DM, Pedreira LC, dos Santos MR, Faustino TN. Concepções da equipe multiprofissional sobre a implementação dos cuidados paliativos na unidade de terapia intensiva. Ciênc Saúde Coletiva. 2013;18(9):2597604. doi: $10.1590 /$ S1413-81232013000900014

6. Brasil. Ministério da Saúde. Diretrizes metodológicas. Brasília: Ministério da Saúde; 2012. 
7. Visser M, Deliens L, Houttekier D. Physician-related barriers to communication and patient- and family-centred decision-making towards the end of life in intensive care: a systematic review. Crit Care. 2014;18(6):604. doi: 10.1186/ s13054-014-0604-z

8. dos Santos MA, Hormanez M. Atitude frente à morte em profissionais e estudantes de enfermagem: revisão da produção científica da última década. Ciênc Saúde Coletiva. $2013 ; 18(9): 2757-68$. doi: $10.1590 /$ S141381232013000900031

9. Freitas NO, Pereira MVG. Percepção dos enfermeiros sobre cuidados paliativos e o manejo da dor na UTI. O Mundo da Saúde. 2013; 37(4):450-7. doi: 10.15343/01047809.2013374450457

10. Ho LA, Engelberg RA, Curtis JR, Nelson J, Luce $J$, Ray DE et al. Comparing clinician ratings of the quality of palliative care in the intensive care unit. Crit Care Med. 2011 ;39(5):975-83. doi: 10.1097/ CCM.0b013e31820a91 db

11. Glavan BJ, Engelberg RA, Downey L, Curtis JR. Using the medical record to evaluate the quality of end-of-life care in the intensive care unit. Crit Care Med. 2008;36(4):1 138-46. doi: $10.1097 / C C M .0 b 013 e 318168 f 301$

12. Curtis JR, Nielsen EL, Treece PD, Downey L, Dotolo D, Shannon SE et al. Effect of a quality-improvement intervention on end-of-life care in the intensive care unit. Am J Respir Crit Care Med. 2011 ; 183(3):348-55. doi: 10.1164/ rccm.201006-10040C

13. Batista KT; Seidl EMF. Estudo acerca de decisões éticas na terminalidade da vida em unidade de terapia intensiva. Com Ciências Saúde. 2011 ;22(1):51-60.

14. Moritz RD, Deicas A, Rossini JP, da Silva NB, do Lago PM, Machado FO. Percepção dos profissionais sobre o tratamento no fim da vida, nas unidades de terapia intensiva da Argentina, Brasil e Uruguai. Rev Bras Ter Intensiva. 2010;22(2):125-32. doi: 10.1590/S0103$\underline{507 \times 2010000200005}$

15. Mendes JA, Lustosa MA, Andrade MCM. Paciente terminal, família e equipe de saúde. Ver SBPH. 2009; 12(1):151-73. 